\title{
COLOMBIA Y LA CONVENCIÓN DE NACIONES UNIDAS SOBRE EL DERECHO DEL MAR: ANÁLISIS JURÍDICO FRENTE AL DILEMA DE LA RATIFICACIÓN
}

\author{
COLOMBIA AND THE UN CONVENTION ON THE LAW OF THE \\ SEA: LEGAL ANALYSIS REGARDING THE DILEMMA OF THE \\ RATIFICATION
}

\author{
GiovanNy Vega-Barbosa* \\ STEFfany Serebrenik-Beltrán** \\ María Camila Aponte-MarTínez***
}

\begin{abstract}
RESUMEN: Este artículo aborda las cuestiones jurídicas asociadas a la eventual ratificación de la Convención de Naciones Unidas sobre el Derecho del Mar por parte de Colombia. Los autores sostienen que la mayoría de preocupaciones que otrora desmotivaron la ratificación de la Convención se han desvanecido de manera progresiva. Sin embargo, desaconsejan de manera transitoria la manifestación del consentimiento internacional a la luz del actual reclamo de derechos históricos el Archipiélago de San Andrés.
\end{abstract}

Palabras clave: Colombia, ratificación, CONVEMAR, derechos históricos de pesca.

ABSTRACT: This article addresses the legal issues arising out of the potential ratification of the UN Convention on the Law of the Sea by Colombia. The authors posit that the majority of the concerns previously deterring Colombia from ratification have progressively disappeared. Yet, they transitorily discourage ratification due to Colombia's current claim of historic rights in the San Andrés Archipelago.

Keywords: Colombia, ratification, LOS Convention, historic fishing rights.

\footnotetext{
* Profesor titular de la cátedra de Derecho Internacional Público y Codirector del Proyecto de Investigación sobre el Derecho del Mar de la Universidad del Rosario. Coordinador del Grupo Consultivo de la Dirección de Asuntos Jurídicos Internacionales del Ministerio de Relaciones Exteriores de Colombia entre el año 2012 y 2015. Correo electrónico: giovanny.vega@urosario.edu.co. Dirección postal: Carrera 7a \# 135 - 78 Bogotá, Colombia.

** Abogada con profundización en derecho internacional de la Universidad del Rosario e investigadora senior del Proyecto de Investigación sobre el Derecho del Mar de la Universidad del Rosario. Correo electrónico: steffany.serebrenik@urosario.edu.co; Calle 115 No. 9-20 Bogotá, Colombia.

*.. Abogada especialista en Derecho Administrativo de la Universidad del Rosario e investigadora senior del Proyecto de Investigación sobre el Derecho del Mar de la Universidad del Rosario. Correo electrónico: maria. aponte@urosario.edu.co; Transversal 23 No. 93-51 Bogotá, Colombia.
} 


\section{INTRODUCCIÓN}

El 10 de diciembre de 1982, la República de Colombia suscribió la Convención de Naciones Unidas sobre el Derecho del Mar (la Convención o la CONVEMAR) ${ }^{1}$. Pese a su protagónica y exitosa participación en la Tercera Conferencia sobre el Derecho del Mar (la Tercera Conferencia) ${ }^{2}$, Colombia no ratificó la Convención y por ende, no le es jurídicamente vinculante como tratado. La firma del instrumento internacional por parte de Colombia lo distingue de otros Estados que también fueron partícipes notables en la Tercera Conferencia, pero se decidieron por un voto en contra de su texto, entre ellos, Estados Unidos de América (EUA) ${ }^{3}$ y Venezuela ${ }^{4}$. La principal diferencia es de carácter jurídico. En virtud del artículo 18 de la Convención de Viena sobre el Derecho de los Tratados de $1969^{5}$, la firma de la Convención obliga a Colombia a abstenerse de llevar a cabo actos que frustren su objeto y fin.

El reconocimiento de la Convención como la constitución de los mares ${ }^{6}$ y la extensión de los derechos marítimos de Colombia por más de $928.600 \mathrm{~km}^{2}$, contrasta con una escasa producción académica en relación con las consideraciones de orden jurídico que justificarían o deberían desmotivar la eventual ratificación ${ }^{7}$. Pese a lo anterior, los actuales litigios entre Nicaragua y Colombia ante la Corte Internacional de Justicia (CIJ), así como actuaciones domésticas altamente progresistas entre las que se incluye el establecimiento de la denominada "Zona Contigua Integral" (ZCI), ubican a Colombia en el foco de atención de la comunidad internacional ${ }^{8}$.

El objetivo general del presente esfuerzo académico es aportar una visión jurídica que permita revelar si los obstáculos históricamente invocados como impedimentos para la ratificación constituyen razones fundadas o, por el contrario, carecen de soporte legal. De esta forma, este escrito no aborda (i) los beneficios económicos y de otra índole que derivarían para Colombia como consecuencia de la ratificación; (ii) la medida en que esos beneficios podrían ser alcanzados por fuera del régimen legal previsto en la Convención; (iii) ni

\footnotetext{
1833 UNTS 3. El texto de la Convención fue adoptado el 30 de abril de 1982 en la ciudad de Nueva York por 130 votos a favor, 17 abstenciones y 4 votos en contra (Israel, Turquía, Estados Unidos y Venezuela). La Convención entró en vigor internacional el 16 de noviembre de 1994. El periodo de firma se extendió hasta 1984 fecha en la que se verificó la suscripción por parte de 155 Estados. Ver en: Millicay (2015) p. 280.

2 Uribe (2001) p. 34; Ferrero (2013) p. 383-410; Llanos (1991) p. 279.

MiLes (1998) p. 36.

JAGOTA (1985) p.57.

1155 UNTS.

KoH (1983) p. xxiii. En el mismo sentido, Scovazzi (2000) p. 122.

Monroy (2006); CAdena y Pariño (2013) p. 61-90.

8 Ringbom (2015) p. 265; Busch (2016) p. 159; Mossop (2017) p. 78; Uchkunova (2016); Vega (2016); Guilfoyle (2012).

9 Recientemente, el Consejo de Relaciones Exteriores emitió su Reporte sobre "Imperativos Árticos", en el que recomendó a los Estados Unidos de América la adhesión a la CONVEMAR. Como el presente escrito, el Reporte favorece un análisis normativo, por sobre consideraciones de índole económico o de logro de objetivos relevantes por fuera del marco de la Convención. Allen et al. (2017).
} 
tampoco las cargas financieras, procesales y reglamentarias que trae aparejada la entrada en vigor de la CONVEMAR.

En el desarrollo del presente trabajo se formularon tres hipótesis principales. (1) En primer lugar, que dada la vastedad de la Convención y la posibilidad de discernir preocupaciones específicas de alto nivel para el Estado colombiano, el análisis de conveniencia frente a la ratificación debería evaluarse a partir de un enfoque preponderantemente inductivo, casuístico y pragmático. (2) En segundo lugar, que las objeciones históricas a la manifestación del consentimiento internacional estuvieron fundadas en preocupaciones de carácter jurídico, por el eventual reconocimiento de estándares desfavorables en conflictos pendientes de resolución con los vecinos marítimos del Estado colombiano. (3) En tercer lugar, que en la actualidad esas preocupaciones han perdido vigencia como consecuencia de dos consideraciones principales: (3.1) la consolidación de las normas concernidas de la Convención en normas de derecho internacional consuetudinario; (3.2) la práctica y actos jurídicos unilaterales del Estado colombiano frente a algunas de estas.

A la luz de las hipótesis planteadas, el presente trabajo se divide en tres partes principales.

La primera de ellas contiene una descripción de la aproximación de la academia colombiana a la cuestión de la ratificación de la CONVEMAR. Por constituir un referente obligado y un eje de contraste, en esta sección se incluye además un análisis sucinto de las discusiones que en EUA se han generado frente a este mismo problema jurídico. La segunda parte contiene el análisis de los autores frente al desvanecimiento de los problemas jurídicos que otrora hubieran podido explicar la reticencia a la ratificación y las razones que explican este fenómeno. La parte final de esta sección aborda el reclamo de derechos históricos en el Archipiélago de San Andrés por parte de Colombia, dentro del litigio sobre las Presuntas Violaciones de Derechos Soberanos y Espacios Marítimos de Nicaragua ante la CIJ. Los autores consideran que los desarrollos recientes de la jurisprudencia internacional, en particular, el Laudo de 12 de julio de 2016 en el Arbitramento del Mar del Sur de Chi$\mathrm{na}^{10}$, desaconsejan la ratificación de la Convención hasta tanto la CIJ se pronuncie sobre el mérito del reclamo colombiano. La tercera parte contiene las conclusiones de los autores.

El presente escrito hace parte de los desarrollos logrados por el Proyecto de Investigación sobre el Derecho del Mar de la Universidad del Rosario.

\section{LA APROXIMACIÓN DOCTRINAL COLOMBIANA A LA CUESTIÓN DE LA RATIFICACIÓN Y EL CONTRASTE CON LA APROXIMACIÓN DE EUA}

Los tratadistas colombianos fueron protagonistas del proceso de gestación del régimen jurídico de los océanos tal y como lo conocemos. Ya en la Conferencia de Codificación de La Haya de 1930, Jesús María Yepes había presentado un importante estu-

\footnotetext{
10 Matter of the South China Sea Arbitration before an Arbitral Tribunal constituted under Annex VII to the 1982 United Nations Convention on the Law of the Sea between the Republic of the Philippines and the People's Republic of China, PCA Case No 2013-19 (The South China Sea Arbitration. Philippines v. China).
} 
dio sobre la noción de líneas de base recta ${ }^{11}$, la cual precedió por dos décadas la primera aproximación de la CIJ al asunto ${ }^{12}$. En la Primera Conferencia sobre el Derecho del Mar, las intervenciones del Profesor José Joaquín Caicedo Castilla fueron fundamentales en las discusiones sobre la línea media de delimitación, la noción de "circunstancias especiales", así como en la progresiva construcción de la noción de solución de disputas a través de un mecanismo pacífico pero jurídicamente obligatorio ${ }^{13}$. El 2 de febrero de 1972, durante la Segunda Conferencia sobre el Derecho del Mar, Alfredo Vázquez Carrizosa presentó un proyecto sobre el denominado "Mar Patrimonial", con una extensión de 200 millas náuticas. En la Tercera Conferencia, en voz del Profesor Joaquín Gori, Colombia consolidó su oposición a la noción ginebrina de isla, insistiendo en la necesidad de diferenciar entre aquellas capaces de mantener habitación humana o una vida económica propia ${ }^{14}$. También defendió el reconocimiento de un estatus jurídico especial para los archipiélagos de Estado y, con ocasión de los Fallos de la CIJ de 1969 sobre la Plataforma Continental del Mar del Norte $^{15}$, se opuso a la consolidación de la noción de circunstancias especiales como norma convencional. Finalmente, Colombia resultaría triunfante en su defensa, compartida con Estados Unidos, Países Bajos y Australia, de una fórmula integrada, flexible pero obligatoria de resolución de controversias ${ }^{16}$.

Pese a esta notable participación en las tres conferencias sobre el derecho del mar, lejos de analizar la cuestión de la ratificación de la CONVEMAR, los tratadistas colombianos se aproximaron al nuevo derecho del mar de manera periférica y con ocasión de las controversias históricas con los vecinos marítimos. Un primer grupo de autores abordó los aspectos jurídicos e históricos del diferendo territorial y limítrofe con la República de Nicaragua $^{17}$. Un segundo grupo abordó la disputa territorial y marítima con la República de Venezuela haciendo un especial énfasis en la controversia territorial frente a las islas "Los Monjes" 18 , así como en las dificultades generadas por la imposibilidad de acordar una frontera marítima en el Golfo de Venezuela ${ }^{19}$. Un tercer grupo abordó las dinámicas históricas y los esfuerzos diplomáticos que determinaron la configuración en el Mar Caribe Occiden$\mathrm{tal}^{20}$. Finalmente, en un cuarto grupo encontramos los académicos, los más jóvenes ${ }^{21}$ y los más veteranos ${ }^{22}$, que se aproximaron al estudio del derecho del mar a partir y con ocasión

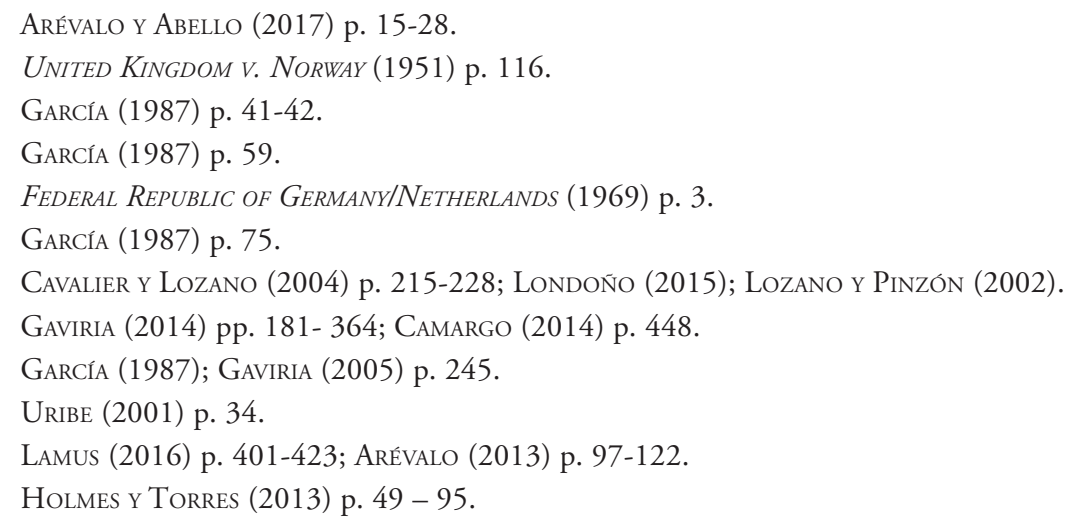


del fallo de 19 de noviembre de 2012 de la CIJ, por el cual se resolvió la Controversia Territorial y Marítima (Nicaragua v. Colombia) ${ }^{23}$.

La limitada producción doctrinal frente a la relación de Colombia con la CONVEMAR podría encontrar explicación en una posición altamente restrictiva y reservada por parte de las autoridades públicas, en relación con las motivaciones del Estado colombiano para abstenerse de ratificar la Convención. Una segunda razón sería consecuencia de la primera pues, en ausencia de claridad acerca de las situaciones y hechos que sustentan el mantenimiento del statu quo frente a la Convención, resulta natural que los esfuerzos académicos se desmotiven al parecer irrelevantes y excesivamente especulativos.

Sin perjuicio de lo anterior, el trabajo de campo de la presente investigación permitió identificar controversias y asuntos inconclusos que con alto grado de uniformidad se invocaron como explicativos de la decisión de no ratificar la CONVEMAR ${ }^{24}$. La Controversia Territorial y Marítima con la República de Nicaragua se ha presentado en distintos foros una de las razones que, durante más de 11 años de litigio, determinó la imposibilidad de avanzar hacia la ratificación ${ }^{25}$. En este punto, los autores infieren que al menos dos interrogantes podrían haber generado disuasión entre los tomadores de decisiones de la época. El primero habría versado, de forma infundada, sobre la metodología de delimitación. En efecto, aunque hoy resulta claro que la CONVEMAR no consagra ningún método para el trazado de la frontera marítima ${ }^{26}$, en aquel momento podría haberse considerado que la CONVEMAR implicaba necesariamente la aplicación de la línea media a la luz de las circunstancias relevantes y, por consiguiente, un alto riesgo para el mantenimiento de la integridad del Archipiélago de San Andrés. Paradójicamente, ante la CIJ Colombia terminaría acogiendo esta metodología de delimitación, en oposición a la metodología de enclaves propuesta por Nicaragua, pero en renuncia al meridiano 82 como frontera marítima. El segundo interrogante habría versado, de forma contradictoria, sobre la distinción prevista en la CONVEMAR entre islas con y sin capacidad para sostener habitación humana o vida económica propia. Como se expondrá en el acápite respectivo, esa distinción fue promovida y liderada por la diplomacia colombiana en la Tercera Conferencia. En ese momento, la atención se encontraba en la controversia inconclusa con la República de Venezuela, en relación con el estatus jurídico de "Los Monjes" y su efecto en la delimitación en la península de la Guajira. Actualmente, las demandas de 2013, presentadas por la República de Nicaragua contra la República de Colombia ante la CIJ, generan nuevamente interrogantes frente a la conveniencia de ratificar la Convención. Con similar contemporaneidad, el

\footnotetext{
23 Nicaragua v. Colombia (2012) p. 624.

24 Al efecto se consultaron textos académicos, comunicados oficiales y notas de prensa. También se sostuvieron conversaciones con funcionarios de las Dirección de Asuntos Jurídicos Internacionales y Soberanía Territorial del Ministerio de Relaciones Exteriores de Colombia, la Comisión Colombiana del Océano, la Dirección General Marítima y el Centro de Investigaciones Oceanográficas e Hidrográficas. Finalmente, se realizaron reuniones con importantes tomadores de decisiones y doctrinantes de la esfera nacional colombiana, entre ellos, ex cancilleres y delegados de Colombia para la Tercera Conferencia sobre el Derecho del Mar.

25 DeVia et al. (2012) p. 122.

26 En su reciente Fallo en el caso de la Delimitación Marítima en el Océano Índico (Somalia v. Kenia), la CIJ recordó que el artículo 83 de la CONVEMAR prevé que la delimitación de las áreas marítimas deberá realizarse de común acuerdo, pero no establece un método específico de delimitación. SOMALIA V. KENIA (2017) para. 90. Ver Cádenas y Herrera (2013) pp. 243-251.
} 
hallazgo del Galeón San José en aguas cercanas a las costas de Cartagena ${ }^{27}$ y las demandas de la empresa Sea Search Armada ${ }^{28}$, son fuente de preocupaciones por los presuntos efectos negativos que algunas disposiciones de la Convención podrían generar en las pretensiones colombianas $^{29}$. Como se verá, la posición de los autores es que las preocupaciones frente al régimen legal aplicable a la eventual recuperación del Galeón San José constituyeron la razón coyuntural que habría desaconsejado la ratificación de la CONVEMAR. Sin embargo, los autores sostienen que solo en fecha reciente, como consecuencia del alegato de derechos históricos de pesca en el Archipiélago de San Andrés, ha emergido un verdadero obstáculo jurídico para la manifestación del consentimiento. Este obstáculo se caracteriza en todo caso como uno de carácter transitorio y contingente.

A la luz de las consideraciones precedentes, los autores concluyen que, en el caso colombiano, la metodología más aconsejable de aproximación a la cuestión de la ratificación debe ser una de carácter preponderantemente inductivo, casuístico y pragmático. En efecto, en el caso colombiano parece claro que existen preocupaciones que, más allá de su reducido desarrollo en la doctrina, influencian de forma definitiva la toma de las decisiones por cuanto versan sobre bienes jurídicos estatales de altísimo valor, a saber, el territorio, la seguridad, la protección medioambiental y el patrimonio arqueológico. Esta forma de aproximación contrasta con una de carácter preponderantemente deductivo, abstracto y dogmático ${ }^{30}$. En esta última, la metodología de valoración implicaría un análisis desprovisto de consideraciones de costo-beneficio y, por lo tanto, aislado de las preocupaciones particulares que generan disuasión frente al otorgamiento del consentimiento internacional. Un proceder de este tipo implicaría en últimas analizar uno a uno los artículos de la Convención, a efectos de definir cuáles de ellos ya vinculan a Colombia por ser declarativos, cristalizadores o constitutivos de la costumbre internacional ${ }^{31}$. Esta faraónica forma de proceder parece inocua y poco ajustada al tipo de valoraciones de conveniencia que preceden las decisiones relevantes de los Estados frente a los tratados ${ }^{32}$.

La posición de los autores encuentra confirmación en la práctica de los EUA, en donde los principales intereses eran reconocibles incluso antes del establecimiento del Comité Ad Hoc de los Fondos Marinos ${ }^{33}$. Esto se debe, entre otras razones, a que versaban sobre asuntos no resueltos durante la Primera y Segunda Conferencia sobre el Derecho del $\mathrm{Mar}^{34}$, y que se mantuvieron visibles a lo largo de la negociación. La publicidad de la posición estatal propició un acompañamiento constante de la academia, la cual persiste al día de hoy y permite pronunciarse con autoridad sobre los intereses que aconsejan o desaconsejan la adhesión de EUA a la Convención.

\footnotetext{
27 El Espectador (4/12/2015): "Santos anuncia hallazgo del Galeón San José".

28 Procuraduría General de la Nación (2008).

29 Mateus-Rugeles (2016) pp. 433-435.

30 En un contexto diferente, las virtudes de un enfoque metodológico mixto o "flexible y fluido" fueron abordadas en el Primer Informe del Relator Especial de la Comisión de Derecho Internacional en relación con el Jus Cogens, señor Dire Tladi.

31 Talmon (2015) pp. 417-443.

32 Roach (2014) p. 239;

33 Dupuy y Vignes (1991) p. 589.

34 Oxman (1985) pp. 4-9.
} 
La posición de EUA en 1973 estuvo altamente influenciada por el "Reporte de la Comisión Stratton", que hizo énfasis en la necesidad de ejercer control regulatorio sobre actividades nocivas para los océanos, así como en la importancia de asegurarle a su armada la capacidad de llevar a cabo misiones de defensa nacional en cualquier lugar de los océanos $^{35}$. Entre los intereses trascendentales se encontraba la preservación de sus libertades de sobrevuelo y navegación frente a los ejercicios de jurisdicción extendida o "creeping jurisdiction" 36 por parte de los Estados costeros que, liderados por Chile y Perú, reclamaban derechos marítimos hasta las 200 millas náuticas ${ }^{37}$.

Una vez en la Tercera Conferencia, la rápida verificación de un apoyo abrumador al reclamo de un mar territorial de 12 millas náuticas derivó en que EUA se concentrara en la negociación de un régimen favorable de paso de tránsito por los estrechos usados para la navegación internacional. Este era un interés de altísima relevancia ya que el reconocimiento de un mar territorial de 12 millas náuticas, sin la aceptación de un correlativo régimen de libertades para terceros Estados, hubiese implicado en la práctica el cierre a la navegación a través de más de 100 estrechos $^{38}$.

En marzo de 1981, Ronald Reagan, como Presidente entrante, impartió instrucciones a su delegación para que el texto de la Parte XI de la Convención fuera renegociado ${ }^{39}$. Pese a esto, ese mismo año se emitió el primer "Proyecto Oficial de la Convención" ${ }^{40}$ y el 30 de abril de 1982 se adoptó su texto definitivo. La doctrina sostiene de forma unánime que EUA votó en contra de la adopción de la Convención y por ende no la suscribió, como consecuencia de sus fuertes reservas a las obligaciones de transferencia de tecnología previstas en la Parte $\mathrm{XI}^{41}$. También se expresa con uniformidad que la versión modificada de la Parte XI fue aceptada por la administración Clinton, situación que explica que EUA firmara el Acuerdo de Aplicación de $1994^{42}$.

En la actualidad, la academia expresa que EUA no ha adherido a la Convención por la imposibilidad de obtener las mayorías necesarias al interior del Senado ${ }^{43}$. Más allá de estas dificultades, hasta el año 2016, el Departamento de Estado mantuvo pública y vigente su intención de adherirse a la CONVEMAR ${ }^{44}$. Se sostiene que una de las razones más fuertes para proceder a la adhesión es la certeza legal que el régimen CONVEMAR genera en el establecimiento del borde exterior del margen continental más allá de las 200 millas náuticas $^{45}$. La elección del Presidente Donald Trump ha generado nuevos desarrollos y preocupaciones entre los teóricos. Aunque los principales tratadistas reconocen que la adhesión

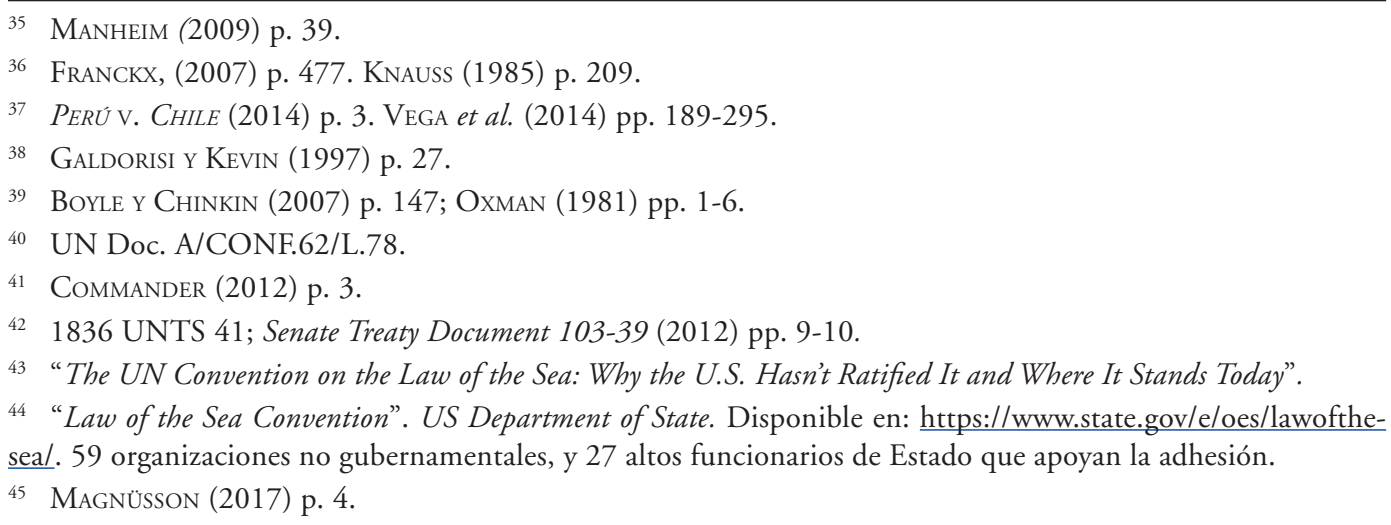


no parece viable en el corto plazo, se han identificado nuevos pasos de cara a la promoción de la CONVEMAR, entre ellos, la construcción de argumentos de costo-beneficio que aborden las preocupaciones de los senadores del occidente medio de EUA que rechazan la adhesión. Estos nuevos argumentos se enfocarían en los riesgos derivados de la explotación de metales críticos del lecho marino por parte de China, las actividades rusas en el ártico y las tensiones en el Mar del Sur de China ${ }^{46}$. Frente a los nuevos desafíos, se estima urgente disponer de legitimidad para reclamar el respeto de los estándares normativos previstos en la CONVEMAR.

Como puede observarse, tanto Colombia como EUA concurrieron a la Tercera Conferencia con intereses definidos. En el caso de EUA, sus intereses se concretaban en normas específicas. En consecuencia, aunque los resultados le fueron altamente favorables, EUA no firmó la Convención por cuanto la Parte XI en particular le resultó manifiestamente inaceptable. El caso de Colombia es más problemático. Como consecuencia de importantes triunfos en relación con intereses de alto calado, Colombia suscribió la CONVEMAR y por lo tanto manifestó de manera inequívoca su acuerdo con el texto final. No obstante, inmediatamente después de la firma surgirían nuevas tensiones que con el paso del tiempo consolidarían una posición temerosa y altamente especulativa frente a los efectos derivados de la ratificación.

\section{EL DESVANECIMIENTO DE LOS PROBLEMAS JURÍDICOS QUE OTRORA HUBIERAN PODIDO INSPIRAR LA DECISIÓN DE NO RATIFICAR LA CONVEMAR}

Las siguientes líneas contienen el análisis de los autores frente a los problemas jurídicos comúnmente invocados como justificantes de la decisión de Colombia de no ratificar la CONVEMAR. Según fue advertido, la posición de los autores es que, como consecuencia de la consolidación de las normas concernidas de la Convención en derecho internacional consuetudinario, o por efecto de actos jurídicos unilaterales del Estado colombiano, la mayoría de los obstáculos históricos se han desvanecido.

\subsection{LA REgUlaCión DE LAS ISLAS EN LA CONVEMAR.}

El numeral $3^{\circ}$ del artículo 121 de la CONVEMAR determina el punto de inicio de la discusión que propone el presente acápite. La norma en comento expresa lo siguiente:

"Régimen de las islas

(...)

3. Las rocas no aptas para mantener habitación humana o vida económica propia no tendrán zona económica exclusiva ni plataforma continental.”

En los términos de la Convención, de la aptitud para mantener la habitación humana o para sostener una vida económica propia, depende la posibilidad de que las islas proyecten derechos marítimos más allá de las 12 millas náuticas. Sin estas cualidades, las

46 Ocean Law News: (30/03/2017) "Changing Strategies for the LOS Convention". 
formaciones naturales de tierra en el mar que sobresalgan en pleamar se ajustarán a la noción de roca, una categoría de islas con derechos limitados ${ }^{47}$.

Según lo explica el ex Canciller colombiano Julio Londoño Paredes, el interés de Colombia en el numeral $3^{\circ}$ del artículo 121 de la Convención estuvo históricamente determinado por su intención de contrarrestar los efectos de las islas "Los Monjes" en una eventual delimitación marítima con la República de Venezuela ${ }^{48}$. Aunque este aspecto de la Convención benefició a Colombia en un primer momento, las recientes controversias contra la República de Nicaragua en el Mar Caribe Occidental le han generado la necesidad de defender el carácter de islas plenas de varias formaciones marítimas del Archipiélago de San Andrés. El fallo de 19 de noviembre de 2012, aunque se pronunció sobre la naturaleza jurídica de formaciones como Quitasueño 32, dejó abierto este interrogante en relación con otras islas que tendrán incidencia en una eventual delimitación con Nicaragua más allá de las 200 millas náuticas desde su costa continental. En este contexto, surge el interrogante de si el numeral $3^{\circ}$ del artículo 21 de la CONVEMAR constituye un obstáculo para la ratificación.

Existe una literatura jurídica abundante que cuestiona el carácter consuetudinario del numeral $3^{\circ}$ del artículo 121 de la CONVEMAR ${ }^{49}$, e incluso su idoneidad para constituir una norma codificadora ${ }^{50}$. Además, la práctica estatal ${ }^{51}$ y judicial ${ }^{52}$ es mínima frente a renuncias o limitación de derechos marítimos a la luz del numeral $3^{\circ}$, y no ofrece guías claras sobre lo que constituye una roca bajo el derecho internacional ${ }^{53}$. Sin perjuicio de lo anterior, en virtud de la conducta del Estado colombiano, estas discusiones parecen estrictamente académicas y no deberían influenciar el juicio de ratificación. En primer lugar, la posición colombiana favoreció y fue decisiva en la inclusión del numeral $3^{\circ}$ precitado en el texto final de la CONVEMAR. Así lo puso de presente el reciente laudo en el caso del Mar del Sur de China ${ }^{54}$. En segundo lugar, las discusiones frente a la oponibilidad del artículo 121 de la CONVEMAR carecen de relevancia práctica pues Colombia ya ha manifestado ante la comunidad internacional que reconoce el carácter consuetudinario de esta norma in totto. Así quedó registrado en el fallo de 19 de noviembre de 2012 de la CIJ55.

\footnotetext{
47 Vega et al. (2015) pp. $399-400$.

48 LONDOÑO (2015) p. 75.

49 KWIATKOWSKa Y SOONS (1993) pp. 139-181.

50 Elferink (1994) p. 37; TANaKa (2012) p. 67.

51 El artículo 51 de la Ley Federal del Mar de los Estados Unidos Mexicanos de 1986 consagra, en los siguientes términos, uno de los pocos casos de consagración normativa doméstica del contenido del artículo 121 (3): "Las islas gozan de zona económica exclusiva pero no así las rocas no aptas para mantener habitación humana o vida económica propia.” Disponible en: http://dof.gob.mx/nota detalle.php?codigo=4781204\&fec $\underline{\mathrm{ha}=08 / 01 / 1986}$.

52 QATAR V. BAHRAIN (2001) pp. 40, 97, para. 185. Ver BOWETt (1979) p. 34.

53 Whomersley (2016) pp. 239-264.

54 Philippines v. China (2016) p. 218, para. 519; "189th Plenary Meeting," UN Doc. A/CONF.62/SR.189, p. 66 at p. 83, para. 251 (8 December 1982).

55 Nicaragua v. Colombia (2012) p. 624, p. 674.
} 
Así las cosas, Colombia debería dirigir sus esfuerzos al cumplimiento de los requisitos previstos en el numeral $3^{\circ}$ supra y no al cuestionamiento del alcance consuetudinario de la norma ${ }^{56}$.

\subsection{INVESTIGACIÓN CIENTÍFICA, PROTECCIÓN DEL MEDIO MARINO Y TRANSFERENCIA DE TECNOLOGÍAS}

A pesar de la muy escasa producción académica en relación con el objeto de análisis, fue posible identificar un documento oficial de la Comisión Colombiana de Oceanografía, hoy Comisión Colombiana del Océano (CCO), el cual puso de presente que entre 1997 y 1998, el Ministerio de Relaciones Exteriores de Colombia convocó a varias entidades concernidas con la implementación de la política marítima, para analizar la conveniencia de ratificar la CONVEMAR. El documento público solo contiene el pronunciamiento de la CCO a favor de la ratificación, en razón de la importancia de los contenidos de las Partes XII (Protección del Medio Marino), XIII (investigación científica) y XIV (Desarrollo y Transmisión de la Tecnología Marina) ${ }^{57}$. Allí se indica que el principal beneficio es el carácter vinculante que conforme a estas normas reviste la investigación científica. La CONVEMAR obliga al Estado a "formular planes, proyectos y programas de investigación, y adoptar y convenir normas, estándares, principios y procedimientos que den soporte científico al resto de las disposiciones objeto de esos tratados." ${ }^{58}$ A su turno, esta situación genera necesidades de creación de nuevas entidades, así como la adecuación, modificación y fortalecimiento de las existentes ${ }^{59}$.

\subsection{El caso sobre la Cuestión de la Delimitación de la Plataforma Continental Más allá de las 200 millas náuticas desde la costa NICARAGÜENSE}

El punto de inicio en este acápite lo constituye el numeral $1^{\circ}$ del Artículo 76 de la Convención, el cual reconoce a los Estados el derecho a una plataforma continental "a todo lo largo de la prolongación natural de su territorio hasta el borde exterior del margen continental”. El artículo 76 de la Convención consagra para sus Estados parte las fórmulas para el establecimiento del borde exterior del margen continental (numeral $4^{\circ}$ ), los limitantes a la línea que fija el punto exterior (numerales $5^{\circ}, 6^{\circ}$ y $7^{\circ}$ ), así como el procedimiento para la delineación del punto exterior del margen continental (numeral $8^{\circ}$ ). Se trata de fórmulas alternativas con miras a maximizar la extensión de la titularidad estatal ${ }^{60}$. Cuando el Estado

\footnotetext{
56 Lo anterior se entiende sin perjuicio de la aquiescencia internacional frente a la naturaleza jurídica de algunas islas en el Océano Pacífico y en el Mar Caribe Occidental.

57 CCO (2000) p. 4.

58 CCO (2000) p. 26.

59 "Un ejemplo de esto son las técnicas y métodos de investigación para la protección del medio marino por la contaminación cuyos resultados dependen de un alto conocimiento especializado en oceanografía química, química marina, biología de la toxicidad, ingeniería sanitaria y derecho ambiental entre otras. Las técnicas y métodos vinculados con la distribución de los contaminantes en el mar, requieren de especialización en oceanografía física, geología marina y de costas, ecología marina y dinámica de poblaciones." CCO (Bogotá, 2000), p. 26.

60 Sмith y Tағт (2000) p. 19
} 
costero considera que el borde exterior de su plataforma continental se ubica más allá de las 200 millas náuticas, este deberá presentar la evidencia que acredita este hecho ante la Comisión de Límites de la Plataforma Continental (CLCS por sus siglas en inglés), la cual emitirá una recomendación sobre la base de la evidencia científica presentada ${ }^{61}$. La Convención prevé que "[l] os límites de la plataforma que determine un Estado ribereño tomando como base tales recomendaciones serán definitivos y obligatorios" ${ }^{\prime 2}$.

El 16 de septiembre de 2013, Nicaragua radicó ante la CIJ una segunda demanda marítima en contra de Colombia relativa a la delimitación de la frontera marítima más allá del límite de las 200 millas náuticas a partir de las líneas de base desde las cuales se mide la anchura del mar territorial de Nicaragua. El 17 de marzo de 2016, la CIJ negó las objeciones preliminares de Colombia, una de las cuales solicitó la inadmisibilidad del reclamo de delimitación más allá de 200 millas náuticas en ausencia de una recomendación por parte de la CLCS $^{63}$. En consecuencia, la CIJ entrará a analizar si Nicaragua efectivamente dispone de derechos sobre la plataforma continental más allá de las 200 millas náuticas.

Frente a este punto debemos recordar que, en el primer capítulo de la trilogía Nicaragua vs. Colombia, la CIJ señaló en el párrafo 126 de su fallo de 19 de noviembre de 2012 que "cualquier reclamación de derechos sobre la plataforma continental más allá de las 200 millas náuticas [por un Estado parte de CONVEMAR] debe estar acorde con el Artículo 76 de CONVEMAR y ser revisado por la CLCS”. Agregó además que, el hecho de que Colombia no sea parte de esta, no releva a Nicaragua de sus obligaciones bajo el Artículo 76 de la Convención ${ }^{64}$. Esta posición constituyó el insumo principal de Colombia para la elaboración de su excepción preliminar contra la admisibilidad de la demanda de 16 de septiembre de 2013 en el segundo capítulo de la trilogía. En este caso, Nicaragua ya había presentado una radicación final ante la CLCS. Sin embargo, invocando el procedimiento CONVEMAR, Colombia objetó la reclamación nicaragüense por considerar que existía una controversia marítima pendiente de resolución. Por disposición expresa del parágrafo 5 (a) del Anexo I de las Reglas de Procedimiento de la CLCS ${ }^{65}$, la objeción colombiana le impidió a la Comisión revisar la pretensión de derechos extendidos de Nicaragua. Para Nicaragua la objeción significó la imposibilidad de cumplir con la condición prevista en el párrafo 126 ya mencionado.

A la luz de este contexto, los autores sostienen que el actual caso de delimitación más allá de las 200 millas náuticas no debería generar disuasión frente a la ratificación de la CONVEMAR. En primer lugar, ante la CIJ Colombia ya ha reconocido que la definición de la plataforma continental prevista en el artículo 76 (1) de la Convención es declarativo de la costumbre internacional ${ }^{66}$. En segundo lugar, a través de la invocación del párrafo 5 (a)

\footnotetext{
61 SuÁrez (2013) p. 345; XueXia (2017) p. 4.

62 NeLson (2009) p. 417.

63 Abello et al. (2017) pp. 609-674.

64 Nicaragua v. Colombia (2012) pp. 624, 668 y 669; Abello y Caro (2012) pp. 219-396.

65 "En caso de que haya una controversia territorial o marítima, la Comisión no considerará ni calificará la presentación hecha por cualquiera de los Estados Parte en esa controversia. No obstante, la Comisión podrá considerar una o varias presentaciones respecto de las zonas objeto de controversia con el consentimiento previo de todos los Estados que sean partes en ella”. CLCS/40/Rev.1 (2008).

66 Nicaragua v. Colombia (2012) p. 624, p. 666.
} 
del Anexo I de la Convención, Colombia ha impedido cualquier posibilidad de que el procedimiento del artículo 76 (8) para el establecimiento del margen continental genere beneficios para Nicaragua. En estos términos, la defensa del Estado Colombia deberá estar dirigida a destacar que, en las actuales condiciones, el ejercicio de delimitación planteado por Nicaragua no es viable pues no se presentan los presupuestos que, en otros casos, han permitido llevar a cabo la tarea de delimitación más allá de las 200 millas náuticas, en ausencia de una recomendación de la Comisión ${ }^{67}$. En todo caso, la argumentación de Colombia ante la CIJ deberá consultar y no afectar de forma desproporcionada sus intereses de largo plazo en el establecimiento de una plataforma continental más allá de las 200 millas náuticas. A este último respecto resulta especialmente relevante la práctica de los EUA y su sometimiento a las fórmulas y restricciones previstas en el artículo 76 de la CONVEMAR ${ }^{68}$.

Sin perjuicio de lo anterior, es pertinente señalar que el caso sub examine reviste una especial relevancia para el derecho del mar contemporáneo ${ }^{69}$. La CIJ deberá decidir, sin precedente previo en el ámbito de la adjudicación internacional, aunque con relevancia para la histórica disputa entre Japón y China en el Mar de China Oriental ${ }^{70}$, si resulta procedente un ejercicio de delimitación que opone títulos independientes sobre la plataforma continental y, a fortiori, si entre estos títulos existen relaciones de prioridad o preeminen$\mathrm{cia}^{71}$. El caso se destaca además por su especial complejidad. En efecto, un pronunciamiento a favor del reclamo nicaragüense supondría que, en una porción de la zona económica exclusiva (ZEE) del Estado colombiano, este perdería el control sobre la explotación de los recursos del lecho y subsuelo marino. Esta posibilidad parecería contradecir la máxima según la cual, no puede existir una ZEE sin una correlativa plataforma continental ${ }^{72}$.

\subsection{El Galeón San José}

Según ha sido ampliamente documentado, el Galeón San José fue hundido el 8 de junio de 1708, tras un enfrentamiento con el navío inglés Expedition en las proximidades de las Islas del Rosario (Colombia) ${ }^{73}$. Tras más de 300 años, el pasado 5 de diciembre de 2015 el Presidente Juan Manuel Santos hizo público el hallazgo del pecio ${ }^{74}$.

El San José, y los problemas jurídicos asociados a su presunto descubrimiento en los primeros años de la década de los 80 del siglo pasado constituyen sin lugar a dudas una de las razones principales, sino la más importante, para que el ímpetu de Colombia en la Tercera Conferencia, y que derivó en la firma de la CONVEMAR, se viera frenado de manera súbita. Aunque no fue posible identificar documentos oficiales que sienten con claridad esta posición, la cronología de los eventos más importantes frente al eventual hallazgo del naufragio permite colegir, con un alto grado de razonabilidad, que Colombia decidió abstenerse de ratificar la Convención al considerar, equivocadamente en juicio de los autores,

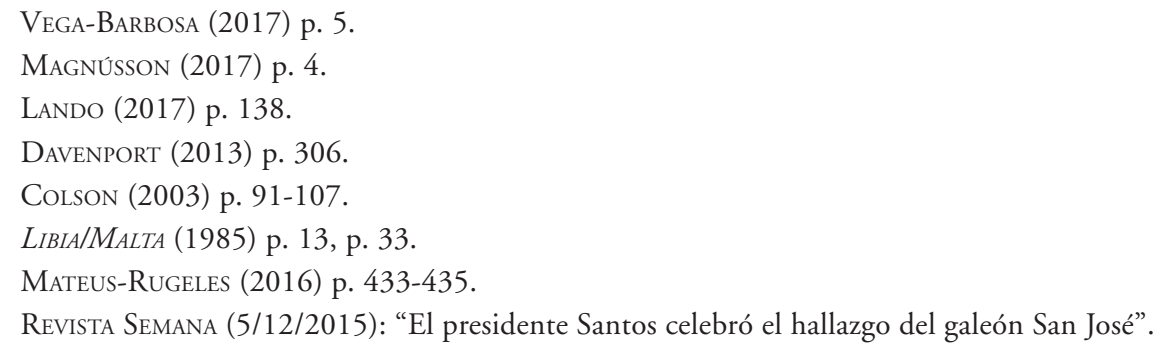


que la definición de espacios y competencias de este instrumento amenazaría el reclamo colombiano sobre el San José.

La primera referencia legal relevante en este análisis es el Decreto 2349 de 1971, mediante la cual se crea la Dirección General Marítima y Portuaria (DIMAR). Conforme a este Decreto, DIMAR expidió la Resolución 0048 de 29 de enero de 1980, por la cual se le otorgó a la compañía estadounidense Glocca Morra Co. Inc. una licencia de dos años para adelantar exploraciones submarinas de búsqueda de antigüedades náufragas en tres áreas específicas ${ }^{75}$. Con autorización de DIMAR, los derechos obtenidos por Glocca Morra Co. Inc., en su calidad de denunciante preferente y beneficiario del primer contrato de exploración fueron transferidos posteriormente a Glocca Morra Co. Esta compañía obtuvo de parte de DIMAR la autorización para explorar nuevas áreas ${ }^{76}$. A pesar del fuerte y constante financiamiento, hasta 1981 Glocca Morra Co. no identificó ningún hallazgo que pudiera asemejarse al San José. Sin embargo, con la adquisición del buque Stage wave, acompañado del submarino August Piccard y un importante equipo técnico y profesional, el 10 de diciembre de 1981 se reportó la localización de un naufragio de dimensiones similares a las del San José ${ }^{77}$. Glocca Morra Co. propuso utilizar un dispositivo de aluminio para extraer material que permitiera establecer mayores conclusiones. DIMAR se opuso a la propuesta dado el evidente riesgo de afectación del patrimonio arqueológico. Por esta razón, se convino que la pieza de aluminio se ubicara encima del hallazgo, en donde sería fácilmente localizable en el futuro. El 18 de marzo de 1982, Glocca Morra Co. entregó un informe confidencial a DIMAR en donde aportó coordenadas y denunció el hallazgo del naufragio. El 3 de junio de 1982, DIMAR expidió la Resolución 0354, mediante la cual reconoció a la sociedad "como denunciante de tesoros en las coordenadas referidas en el Reporte Confidencial sobre Exploración Submarina”. Según lo relata Jorge Bendeck, antiguo miembro de la Comisión de Antigüedades Náufragas, la Secretaría Jurídica de la Presidencia de la República de Colombia estudió varios conceptos de asesores internos y externos en relación con la naturaleza y posibilidad de apropiación de las riquezas arqueológicas localizadas en el fondo del mar colombiano. En una primera parte, los estudios versaron sobre el lugar de ubicación de los bienes, razón por la cual se hacía necesario un estudio sobre el alcance de las instituciones de mar territorial, plataforma continental y de la ZEE. Los conceptos permitieron conocer que la aplicación de la regulación nacional a los objetos ubicados en el lecho marino podría ser problemática más allá de las 12 millas náuticas de mar territorial. Frente a la naturaleza de los bienes, la Secretaría Jurídica acogió la tesis del Profesor Fernando Hinestrosa, según la cual estos son especies náufragas no salvadas y no les resulta aplicable el régimen legal de los bienes mostrencos, sino el de los tesoros ${ }^{78}$. Según lo describe Bendeck, la Secretaría Jurídica concluyó que, al tratarse de tesoros y siempre y cuando se encontraran en el mar territorial o plataforma continental, los bienes eran susceptibles de apropiación ipso jure. De encontrarse en la ZEE, lo procedente sería regular contractualmente los términos de las labores de búsqueda y rescate.

\footnotetext{
Procuraduría General de la Nación (2008) p. 17.

76 Resolución de Dimar No. 0066 (4/2/1981).

77 BENDECK (2003) p. 96.

78 BENDECK (2003) pp. 103-104.
} 
En el concepto de la Secretaría Jurídica de Presidencia, aunque la Ley 10 de 1978 ya limitaba el ejercicio de derechos soberanos en la plataforma continental a los recursos naturales, la ausencia de una restricción similar en la Constitución tenía por efecto, en virtud del principio de primacía normativa, que la soberanía del Estado en la plataforma continental se extendía a toda clase de bienes ${ }^{79}$.

El último episodio de esta primera fase está constituido por la Resolución 204 de 24 de marzo de 1983, mediante la cual DIMAR autorizó la transferencia de los derechos de Glocca Morra Co. a la empresa Sea Search Armada. Con el propósito de confirmar si lo denunciado era el San José, el 7 de septiembre de 1983 esta empresa hizo uso del sumergible Heather Express, el cual efectuó un reconocimiento del lugar en donde se había dejado el objeto metálico. Según lo relata Bendeck, Tom Clayton y Todd Dimitry, abogados de Sea Search Armada, manifestaron que "de estar ubicado fuera de las 12 millas, Colombia no tendría ningún derecho de propiedad de acuerdo con la legislación marítima internacional" $"$.

Con posterioridad a estos hechos se han librado importantes batallas jurídicas en relación con la naturaleza jurídica del naufragio, así como en relación con los derechos de Sea Search Armada ${ }^{81}$.

El presente escrito no pretende agotar la discusión jurídica frente a este punto, y por el contrario constituye una invitación para que el asunto sea objeto de un desarrollo doctrinal profundo. La posición actual de los autores es que el contenido de la CONVEMAR no afecta de manera determinante ninguna de las aristas relevantes en relación con el San José, a saber: la propiedad soberana sobre este y su cargamento, y los derechos de Sea Search Armada. Por lo tanto, el contenido de la CONVEMAR en lo que concierne al patrimonio cultural sumergido no debería constituir un factor decisivo de disuasión para su ratificación por parte de Colombia.

Como bien lo pone de presente la Profesora Andrea Mateus-Rúgeles, en relación con la soberanía sobre el San José, el derecho internacional del mar solo es relevante "a efectos de la ubicación del pecio a partir de las delimitaciones dadas por este derecho" ${ }^{82}$. Para ser más exactos, la regulación de la CONVEMAR frente al patrimonio cultural sumergido está contenida en el artículo 303, del cual es posible extraer 4 conclusiones principales: (i) los Estados en general deben proteger los objetos arqueológicos en el mar y cooperar para ello; (ii) a efectos de fiscalizar el tráfico de estos objetos, es posible presumir que su remoción de la zona contigua sin autorización del Estado costero "constituye una infracción cometida en su territorio o en su mar territorial, de las leyes y reglamentos mencionados en dicho artículo"; (iii) el artículo 303 no produce efectos en relación con los derechos de los propietarios identificables, las normas sobre salvamento, o las leyes sobre intercambios culturales;

79 BENDECK (2003) pp. 101-102.

80 BENDECK (2003) p. 108.

81 Sala de Casación Civil de la Corte Suprema de Justicia (2007); Mediante Oficio confidencial de 2 de noviembre de 1984, y con fundamento en la nueva legislación, el Director General Marítimo y Portuario le indicó al Gerente de Proyectos de Sea Search Armada que, de cara a cualquier contrato de exploración y recuperación, el área de recuperación sería únicamente la descrita por la Compañía Glocca Morra en su Reporte Confidencial de 26 de febrero de 1982. Clarificó además que los únicos derechos de SSA son los cedidos por Glocca y reconocidos por la DIMAR mediante la RESOLUCIÓn 0204 de marzo de 1983.

82 Mateus-Rugeles (2016) p. 433. 
(iv) el artículo 303 se entiende sin perjuicio de acuerdos internacionales específicos sobre protección de patrimonio arqueológico en el mar ${ }^{83}$. Así las cosas, la pretensión de la CONVEMAR frente al patrimonio pareciera ser la de proveer unos mecanismos mínimos de protección en aguas más allá del mar territorial, es decir, en áreas en donde las competencias jurisdiccionales del Estado están limitadas por el texto de la Convención ${ }^{84}$.

En este sentido, como pareciera tenerlo claro Colombia, o al menos así lo sugieren las múltiples reformas normativas y su decisión de no ratificar la Convención de la UNESCO de $2001^{85}$, las respuestas a los interrogantes más relevantes en relación con el San José "girarán entre el derecho de almirantazgo y el del patrimonio cultural sumergido, principalmente" 86 , no alrededor de la CONVEMAR. En todo caso, por cuanto Colombia no es parte ni del Convenio Internacional sobre Salvamento Marítimo de 1989 ni de la Convención UNESCO de 2001, pero por sobre todo, por cuanto la cuestión del San José no se litiga ante cortes domésticas de terceros Estados, la definición de lo que constituye patrimonio cultural sumergido y los derechos que le corresponden a Sea Search Armada deben evaluarse a la luz de su derecho interno ${ }^{87}$ y de los tratados bilaterales aplicables.

Finalmente, los autores estiman relevante advertir que elevar excesivamente el grado de influencia de las disposiciones de la CONVEMAR en la cuestión del San José implica simplificar de forma indebida el objeto de la discusión. Lejos de limitarse a contenidos específicos o exclusivos de la Convención, una eventual controversia interestatal frente al San José versaría sobre la aplicación del principio de intertemporalidad ${ }^{88}$, la naturaleza del Galeón, esto es, si buque de guerra o privado ${ }^{89}$, la aplicación de una teoría de abandono expreso o tácito ${ }^{90}$, la noción de inmunidad de buque de guerra ${ }^{91}$ y su preservación con posterioridad al hundimiento, la relación entre el San José y su cargamento ${ }^{92}$, la aplicación de la teoría de inmunidad relativa en este contexto ${ }^{93}$, entre otros.

\footnotetext{
83 Scovazzi y Garabello (2003) p. 8.

84 Forrest (2010) p. 347.

85 Convención Unesco sobre Patrimonio Cultural Subacuático. Colombia se abstuvo de ratificar la Convención de 2001 en atención a sus preocupaciones por la eventual contradicción con el régimen previsto en la CONVEMAR, el énfasis en la protección in situ, la excesiva protección de los intereses de los Estados del pabellón. Dromgoole (2013) p. 56.

86 Mateus (2016) p. 433.

87 La normativa más reciente a este respecto es la Ley 1675 de 2013, la cual fue objeto de análisis por parte de la Corte Constitucional mediante Sentencia C-572 de 2014. En relación con la posibilidad de que las autoridades judiciales colombianas concluyan que un mismo bien constituye patrimonio cultural de 2 Estados, así como sobre los efectos de una eventual invocación de la inmunidad de ejecución por parte de España; MATEUs y Rodríguez (2016) p.253-275.

88 The Netherlands V. United States of America (1928) pp. 829, 845.

89 SIDAK, The Quasi War Cases-and their Relevance to Whether "Letters of Marque and Reprisal" Constrain Presidential War Powers, at <http://papers.ssrn.com/sol3/papers.cfm?abstract_id=577264>(2005); BEDERMAN (2012) p. 1,4 .

90 SEA HUNT V. UNIDENTIFIED SHIPWRECKED VESSEL (1999), p. 641, 643; RoACH (2012) p. 3.

91 VADI (2010) p.266; VIGNI (2013) p.6.

92 CobB CoIn Co., Inc. v. UNIDENTIFIEd, WRECKEd, ETC (1982); R.M.S. Titanic INC. V. HAVER (1999) p. $967-$ 969; ODYSSEY MARINE V. UNIDENTIFIED SHIPWRECKED VESSEL (2011) p.1180; TREASURE SALVORS, INC. V UNIDENTIFIED WRECKED AND ABANDONED SAILING VESSEL (1978) p. 335-336.

93 GueVara V. PERÚ (2006) p.1289, 1298.
} 


\subsection{LAS PRESUNTAS VIOLACIONES DE LOS DERECHOS SOBERANOS Y ESPACIOS MARÍTIMOS} de Nicaragua en el Mar Caribe Occidental

El 26 de noviembre de 2013, Nicaragua demandó por tercera vez a Colombia ante la CIJ. La controversia en este caso se refiere a la presunta violación de los derechos soberanos de Nicaragua y de sus zonas marítimas, tal y como fueron declaradas en el Fallo de 19 de noviembre de 2012, así como a la alegada amenaza del uso de la fuerza por parte de Colombia con el fin de implementar esas violaciones ${ }^{94}$. Mediante su fallo de 17 de marzo de 2016, en respuesta a las excepciones preliminares propuestas por Colombia, la Corte declaró que únicamente proseguiría al fondo del asunto a efectos de analizar la legalidad del Decreto 1946 de 2013, así como del Decreto 1119 de 2014, modificatorio del anterior, mediante los cuales se prevé y regula la denominada "Zona Contigua Integral” en el Archipiélago de San Andrés 95 .

Nicaragua alega que la ZCI se extiende más allá de las 12 millas náuticas desde el borde exterior del mar territorial de las islas del Archipiélago, e incluye competencias en materia de seguridad que no están autorizadas por el artículo 33 de la CONVEMAR, al igual que otras que como la de control medioambiental, serían exclusivas de Nicaragua como titular de la zona económica exclusiva.

El pasado 16 de noviembre de 2016, un día antes de la presentación de la ContraMemoria de Colombia ante la CIJ, el Ministerio de Relaciones Exteriores informó mediante comunicado de prensa que "una parte importante de la defensa de Colombia en este caso gira alrededor de temas tan trascendentales como (...) los derechos históricos de pesca en las áreas marítimas aledañas al Archipiélago de San Andrés"96. Por esta razón, en este escrito los autores se proponen analizar si, en el caso concreto, una eventual ratificación de la CONVEMAR por parte de Colombia sería contraproducente a su reclamo de derechos históricos.

En un primer momento, es preciso señalar que, en su escrito de excepciones preliminares presentado del pasado 19 de diciembre de 2014, Colombia ya elaboró in extenso sobre la legalidad de sus acciones y de su ZCI. Colombia señaló que bajo el derecho internacional todas sus islas generan zonas contiguas y que estas últimas convergen unas con otras $^{97}$. Agregó que la cuestión de la delimitación de las zonas contiguas no fue abordada por el fallo de $2012^{98}$ y que los decretos 1946 de 2013 y 1119 de 2014 se someten de forma expresa al imperio del derecho internacional y al respeto de los derechos de terceros Es$\operatorname{tados}^{99}$. Frente al contenido de las competencias, Colombia indicó que la seguridad integral del Estado en relación con asuntos como la piratería o el tráfico ilícito de estupefacientes hace parte de las libertades de Alta Mar. Además precisó que solo se asignan competencias de fiscalización en relación con infracciones cometidas en el territorio o en su mar territorial, siendo este el marco de aplicación de sus competencias ambientales ${ }^{100}$. Finalmente,

94 Disponible en: http://www.icj-cij.org/docket/files/155/17978.pdf..

95 Abello et al. (2017) p. 545-608.

96 Comunicado de prensa $(16 / 11 / 2016)$.

97 Nicaragua V. COLOMbia (2010) p. 175, párr. 5.34.

98 Nicaragua v. Colombia (2003) p. 34, párr. 2.50 y ss.

99 Nicaragua v. Colombia (2003) p. 36, párr. 2.55.

100 Nicaragua v. Colombia (2003) p. 38, párr. 2.60. 
Colombia señaló que su ZCI es necesaria para la administración ordenada, el ejercicio de funciones de policía y el mantenimiento del orden público en los espacios marítimos del Archipiélago ${ }^{101}$, criterios estos invocados por la CIJ en su fallo de 19 de noviembre de $2012^{102}$.

Por cuanto la posición del Estado colombiano frente a la zona contigua no sugiere prima facie una separación del régimen previsto en la CONVEMAR, en las siguientes líneas los autores se centrarán en el reclamo de derechos históricos de pesca en el Archipiélago de San Andrés.

En el artículo 7 de la Ley 10 de 1978, Colombia reconoció y proclamó una ZEE. Esta consagración normativa podría determinar, en opinión de algunos estudiosos del derecho internacional, que Colombia ya habría perdido sus derechos históricos de pesca frente a los derechos soberanos de un tercer Estado sobre los recursos vivos de su zona económica exclusiva $^{103}$. Con un propósito eminentemente académico y sin que por ello se comprometa o prejuzgue su posición frente a este particular, la tesis de los autores es que existe al menos un argumento legal plausible que permitiría sostener que, a pesar de su proclamación de una ZEE, la decisión de no ratificación de la CONVEMAR le permite a Colombia seguir alegando derechos históricos de pesca en la ZEE de un tercer Estado.

Tres premisas sustentarían el argumento colombiano.

En primer lugar, la regulación de la ZEE en la CONVEMAR se reconoce como el producto de un delicado balance entre los derechos de los Estados costeros y los de terceros Estados ${ }^{104}$. Además, se reconoce que ese balance es predicable de la totalidad del texto de la Convención y es causa eficiente de los beneficios y restricciones allí incluidos ${ }^{105}$. Por lo tanto, ab initio resulta posible argüir que las relaciones que en la CONVEMAR se establecen entre esta y los derechos históricos es exclusiva de este tratado. En otras palabras, parece por lo menos viable un planteamiento según el cual, la renuncia a los derechos históricos no es consecuencia de la ZEE sino del balance de derechos y obligaciones que se prevén en la CONVEMAR y que, en conjunto, justificarían esa afectación. En segundo lugar, la extinción de los derechos históricos como consecuencia del reconocimiento de la ZEE en la CONVEMAR es el resultado de ejercicios interpretativos propios del derecho de los tratados que llevan a concluir que, por cuanto la CONVEMAR reguló de forma expresa los únicos derechos históricos protegidos, la inexistencia de una cláusula de salvaguarda semejante dentro de la sección $\mathrm{V}$ de la Convención, permite colegir que no fue la intención de los Estados preservar esos derechos cuando se ejerzan en áreas localizadas en la zona económica exclusiva de un tercer Estado. Una línea similar de análisis llevó al Tribunal Arbitral en el caso del Mar del Sur de China a afirmar que, la adhesión de China a la CONVEMAR tuvo por efecto la derogación de los derechos históricos que esta hubiera podido tener sobre los recursos vivos y no vivos en la zona económica exclusiva de Filipinas ${ }^{106}$. En tercer lugar, la práctica demuestra que los Estados que proclamaron zonas económicas ex-

101 Nicaragua v. Colombia (2003) p. 39, párr. 2.63.

102 Nicaragua v. Colombia (2003) p. 708, párr. 230 y p. 716, párr. 244.

103 Kopela (2017) pp. 183-184.

104 Davenport y Beckman (2012) p. 16.

105 JUdA (1986) p. 44.

106 Philippines V. ChINA 2016) p. 111, párr. 262. 
clusivas de manera previa a la entrada en vigor de la CONVEMAR establecieron regímenes que difieren del contenido de esta última ${ }^{107}$. Lo anterior sugiere entonces, al menos prima facie, que un Estado podría reconocer y proclamar en su derecho interno una ZEE, pero abstenerse de ratificar la CONVEMAR al estar en desacuerdo con el arreglo específico previsto en el instrumento, siendo uno de ellos la extinción de sus derechos históricos en áreas marinas bajo jurisdicción de otros Estados.

Así las cosas, para los autores resulta plausible alegar que la renuncia a los derechos históricos por efecto del régimen de ZEE solo opera como consecuencia del reconocimiento del particular balance establecido en la CONVEMAR. Es decir, que la extinción es resultado de la ratificación de esta lex specialis mas no de la proclamación de la ZEE.

Ahora bien, una razón adicional inclinaría la balanza en contra de la ratificación de la CONVEMAR como consecuencia del reclamo de derechos históricos de pesca. En el caso del Mar del Sur de China, el Tribunal distinguió entre la noción genérica de derecho histórico, y la subespecie "título histórico". Para el Tribunal, la primera categoría se refiere a cualquier prerrogativa que nace como consecuencia del paso del tiempo y la aquiescencia de los terceros Estados La segunda categoría constituye una forma específica de derecho histórico que equivalen a un reclamo de soberanía ${ }^{108}$. A continuación, el Tribunal analizó el contenido de la cláusula de exclusión prevista en el artículo 298 de la CONVEMAR y concluyó que solo las disputas sobre títulos históricos pueden ser excluidas de la competencia de los órganos de solución de controversias. Es decir, de seguirse la posición del tribunal arbitral, al momento de ratificar la CONVEMAR Colombia no podría excluir su reclamo de derechos históricos de la competencia de un tribunal internacional establecido conforme a la Convención, pues sus derechos históricos de pesca no equivalen a reclamos de soberanía en los espacios marítimos jurisdiccionales de un tercer Estado ${ }^{109}$.

\section{CONCLUSIÓN}

El dilema de la ratificación de la CONVEMAR por parte de Colombia es aún hoy una tarea pendiente de ser abordada exhaustivamente. Frente a esta importante cuestión, el presente escrito constituye un inicio de aproximación cuyo objetivo es propiciar una aproximación académica más entusiasta a los problemas jurídicos aquí identificados.

El presente estudio pudo constatar las tres hipótesis de investigación planteadas por los autores. En primer lugar, el análisis de la ratificación de la CONVEMAR a partir de un enfoque inductivo, casuístico y pragmático permitió abordar el objeto de investigación en función de los intereses reales del Estado colombiano. También fue posible comprobar que las preocupaciones históricas se enmarcaron en interrogantes de carácter jurídico. Con especial valor para el objeto de estudio, pudo establecerse que los principales logros de Colombia en la Tercera Conferencia estuvieron motivados por la gravedad de la controversia pendiente con Venezuela y, sin embargo, a menos de 10 años de suscrita la Convención,

107 Grbec (2014) p. 239; Rothwell et al. (2015) p. 164; Oceans Act (S.C. 1996, c. 31); Act No. 411 (22/05/1996); "Presidential Proclamation No. 5030 on the Exclusive Economic Zone of 10 March 1983", INTERNATIONAL LEGAL MATERIALS (1980), p. 465.

108 Philippines V. China 2016) p. 96, párr. 225-227.

109 Philippines v. China 2016) p. 313, párr. 801. 
generaron disuasión por los efectos negativos del artículo 121 (3) de la CONVEMAR en la controversia de delimitación marítima con Nicaragua. Finalmente, fue posible aportar evidencia sobre el carácter aparente de algunos de los obstáculos históricamente identificados, y del desvanecimiento de otros como consecuencia de la evolución del derecho internacional o de la conducta del Estado colombiano. En estos términos, el único obstáculo real para la ratificación parece haber surgido en fecha reciente como consecuencia del alegato de derecho históricos de pesca en el Archipiélago de San Andrés.

\section{BIBLIOGRAFÍA CITADA}

Abello, Ricardo et al. (2012): "Traducción del Fallo de la Corte Internacional de Justicia en el "Diferendo Territorial y Marítimo", Anuario Colombiano de Derecho Internacional, vol. 5 (1): pp. 219-396.

Abello, Ricardo et al. (2017): "Traducción de la sentencia de la Corte Internacional de Justicia en el caso: Presuntas violaciones de derechos soberanos y espacios marítimos en el mar Caribe (Nicaragua c. Colombia). Excepciones preliminares", Anuario Colombiano de Derecho Internacional, vol. 10 (1): pp. 545-609.

Abello, Ricardo et al. (2017): “Traducción de la sentencia de la Corte Internacional de Justicia en el caso: Cuestión de delimitación de la plataforma continental entre Nicaragua y Colombia más allá de las 200 millas náuticas desde la costa nicaragüense (Nicaragua c. Colombia). Excepciones preliminares", Anuario Colombiano de Derecho Internacional, vol. 10 (1): pp. 609-674.

Abello, Ricardo \& Arévalo, Walter (2017): “The Influence of Latin-American doctrine on International Law: The Rise of Latin American Doctrines at the Hague Academy during the Early Twentieth Century" en Sorel, Jean-Marc \& Wojciklewicz Paula (Edit.), Latin America and the International Court of Justice: Contributions to International Law (Reino Unido, Routledge).

ArÉvalo, Walter (2013): "El Fallo sobre San Andrés: El debate de la supremacía del derecho internacional, la obligatoriedad del fallo y el derecho interno constitucional colombiano” en Holmes Trujillo Carlos \& Torres María Lucía (Edit.), Contribución de la Universidad del Rosario al debate sobre el fallo de La Haya: Análisis del caso Nicaragua vs. Colombia (Bogotá, Editorial Universidad del Rosario).

Allen, Thad W et al. (2017): Arctic Imperatives: Reinforcing US Strategies on America's Fourth Coast - Independent Task Force Report No. 75 (New York, Washington D.C., CFR).

Bederman, David J (2012): "Privateering” en Max Planck Encyclopedia of Public International Law (Oxford, Oxford University Press).

Bendeck-Olivella, Jorge (2003): El Galeón Perdido ¿Dónde está el San José? (Bogotá, Villegas Editores).

Boyle, Alan \& Chinkin, Christine (2007): The Making of International Law (Oxford, Oxford University Press).

BoweTt, Derek (1979): The Legal Regime of Islands in International Law (Oceana, New York, 1979). 
Busch, Signe Veirud (2016): Establishing Continental Shelf Limits Beyond 200 nautical miles by the Coastal State (Leiden, Brill Nijhoff).

Cadena-Afanador, Walter René \& Pariño-Repizo, Angélica María (2013): "La Convención del Mar y el Conflicto Nicaragua-Colombia sobre el Archipiélago de San Andrés", Revista Republicana, vol. 15: pp. 61-90.

Camargo, Pedro Pablo (2014): Tratado de Derecho Internacional Público (Bogotá, Leyer, Quinta edición).

Cárdenas, Fabián \& Herrera, Viviana (2013): "Una dosis de subjetividad para nada sorpresiva o innovadora: la equidad y el caso Nicaragua Vs. Colombia ante la CIJ", International Law, vol. 22: pp. 243-251.

Cavalier, Germán \& Lozano Simonelli, Alberto (2004): "El ataque de Nicaragua a la soberanía colombiana - ¿Controversia internacional o violación del ius cogens?”, Entre lo Global y lo Local, vol. 70: pp. 215-228;

Commission on the Limits of the Continental Shelf: "Chronology of the Rules of Procedure" CLCS/40/Rev.1 (17 de abril de 2008).

Comisión Colombiana de Oceanografía (2000): Análisis de la incidencia para Colombia al ratificar la Convención de las Naciones Unidas sobre el Derecho del Mar, de 1982, en lo relativo a las Partes XII (Protección del Medio Marino), XIII (investigación cientifica) y XIV (Desarrollo y Transmisión de la Tecnología Marina)" (Bogotá, Comisión Colombiana de Oceanografía.

Commander, Robert C. (2012): "At what cost? America's UNCLOS Allergy in the time of 'Lawfare", Naval Law Review, vol. 61 (1): pp. 3.

Colson, David A. (2003): "The Delimitation of the Outer Continental Shelf between Neighboring States", The American Journal of International Law, vol. 97(1): pp. 91-107.

Davenport, Tara \& BecKman, Robert (2012): "The EEZ Regime: Reflections after 30 years", en Scheiber, Harry (Edit.), Securing the Oceans for the Next Generation (Seoul Korea, Papers from the Law of the Sea Institute, UC Berkeley-Korea Institute of Ocean Science and Technology Conference).

Davenport, Tara (2013): “The China-Japan Dispute Over Entitlement in the East China Sea: Legal Issues and Prospects for Resolution” en Schofield, Clive et al. (Edit.), The Limits of Maritime Jurisdiction (Martinus Nijhoff Publishers).

Devia-Garzon, Camilo et al. (2012): "Colombia y la Tercera Conferencia de Naciones Unidas sobre el Derecho del Mar: un análisis desde la sociedad internacional”, Revista Republicana, vol. 13: p. 122.

Dupuy, René Jean \& Vignes, Daniel (1991): A Hanbook on the New Law of the Sea (Dordrecht, Boston, Lancaster, Martinus Nijhoff Publishers).

ElferinK, A.G.O. (1994): The Law of Maritime Boundary Delimitation: A Case Study of the Russian Federation (Dordrecht, Boson, London, Martinus Nijhoff Publishers).

Ferrero-Costa, Eduardo (2013): "Latin America and the Law of the Sea", en Scheiber, Harry (Edit.) Regions, Institutions, and Law of the Sea Studies in Ocean Governance (Leiden, Boston, Martinus Nijhoff Publishers).

Dromgoole, Sara (2013): Underwater Cultural Heritage and International Law (Cambrdige, CUP). 
Forrest, Craig (2010): International Law and the Protection of Cultural Heritage (London, New York, Routledge).

FranCKX, Erik (2007): "The 200-Mile Limit: Between Creeping Jurisdiction and Creeping Common Heritage?”, The George Washington International Law Review, vol. 39(3): pp. 477.

García, Andelfo J. (1987): La Delimitación de Espacios Oceánicos: El caso Colombo-Venezolano (Bogotá, Universidad Externado de Colombia,).

Gaviria-Liévano, Enrique (2014): Politica Exterior Colombiana. Los Monjes en el Diferendo con Venezuela (Bogotá, Universidad Externado de Colombia).

Galdorisi, George V \& Vienna, Kevin R. (1997): "Beyond the Law of the Sea - New Directions for U.S. Oceans Policy (Connecticut, London, Praeger, Wesport,).

Grbec, Mitja (2014): Extension of Coastal State Jurisdiction in Enclosed and Semi-Enclosed Seas. A Mediterranean and Adriatic Perspective (London, New York, Routledge).

Green, J.A. The Persistent Objector Rule in International Law (Oxford, Oxford University Press).

Guilfoyle, Douglas (2012): "Nicaragua v Columbia: the curious question of the Commission on the Limits of the Continental Shelf", EJIL Talk. Disponible en https://www. ejiltalk.org/nicaragua-v-columbia-the-curious-question-of-the-commission-on-the-limits-of-the-continental-shelf/. Fecha de consulta: 12 de febrero de 2017.

Holmes-Trujillo, Carlos \& Torres, María Lucía (2013): Contribución de la Universidad del Rosario al debate sobre el fallo de La Haya: Análisis del caso Nicaragua vs. Colombia (Bogotá, Editorial Universidad del Rosario).

JaGota, S.P. (1985): Maritime Boundary (Dordrecht, Martinus Nijhoff Publishers).

JudA, Lawrence (1986): "The exclusive economic zone: Compatibility of national claims and the un convention on the law of the sea", Ocean Development \& International Law, vol. 16 (1): pp. 1-58.

Knauss, J.A. (1985), “Creeping Jurisdiction and Customary International Law”, Ocean Development \& International Law, vol. 15: pp. 209.

Kon, T.B. (1983): "A Constitution for the Oceans", UN The Law of the Sea - Official Text of the United Nations Convention on the Law of the Sea with Annexed and Index (New York, United Nations).

Kopela, Sophia (2017): "Historic Titles and Historic Rights in the Law of the Sea in the Light of the South China Sea Arbitration", Ocean Development \& International Law, vol. 48 (2): pp. 183-184.

Kwiatkowska, B. \& Soons, A.H.A: "Entitlements to Maritime Areas of Rocks Which Cannot Sustain Human Habitation or Economic Life of Their Own", Netherlands Yearbook of International Law, vol. 21: pp. 139-181.

Lando, Sophia (2017): "Delimiting the Continental Shelf Beyond 200 Nautical Miles at the International Court of Justice: The Nicaragua v. Colombia Cases", Chinese Journal of International Law, vol. 16 (2): pp. 137-173.

Londoño-Paredes, Julio (2015): Colombia en el Laberinto del Caribe (Bogotá, Universidad del Rosario). 
Lozano-Simonelli, Alberto \& Pinzón-López, Jaime (2002): San Andrés y Providencia. La Amenaza de Nicaragua. Aspectos Jurídicos y Politicos de la Posición de Colombia (Bogotá, Universidad Tadeo Lozano).

Llanos-Mansanilla, Hugo (1991): La Creación del nuevo derecho del mar: el aporte de Chile (Santiago, Editorial Jurídica de Chile).

Magnússon, Bjarni Más (2017): "Can the United States Establish the Outer Limits of Its Extended Continental Shelf Under International Law?”, Ocean Development \& International Law, vol. 48(1).

Manheim, Frank T (2009): The Conflict over Environmental Regulation in the United States Origins, Outcomes and Comparison with the EU and Other Regions (New York, Springer).

Mateus-Rugeles, Andrea (2016): "Comentarios del Observatorio de Derecho Internacional en relación con el Galeón San José”, Anuario Colombiano de Derecho Internacional, vol. 9: pp. 433-435.

Mateus-Rugeles, Andrea \& Rodríguez Valenzuela, Ingrid (2016): "Pecios españoles en aguas colombianas: conjugación entre el patrimonio cultural subacuático y la inmunidad jurisdiccional de los Estados", Revista de Derecho de Valdivia, vol. 29 (2): pp. 253275.

MillicaY, María Fernanda (2015): “The Common Heritage of Mankind: $21^{\text {st }}$ Century Challenges of a Revolutionary Concept" en Del Castillo, Lilian (ed.), Law of the Sea, From Grotius to the International Tribunal for the Law of the Sea-Liber Amicorum Judge Hugo Caminos (Leiden, Boston, Brill Nijhoff).

Monroy-Cabra, Marco Gerardo (2006): El diferendo entre Colombia y Nicaragua sobre el Archipiélago de San Andrés y Providencia (Bogotá, Academia Colombiana de Jurisprudencia).

Mossop, Joanna (2017): The Continental Shelf Beyond 200 Nautical Miles: Rights and Responsibilities (Oxford, Oxford University Press).

Nelson, L.D.M. (2009): 'The Settlement of Disputes Arising from Conflicting Outer Shelf Claims"' International Journal of Marine and Coastal Law, vol. 409: pp. 417.

Procuraduría General de la Nación (2008): El Galeón San José - Patrimonio Cultural Sumergido - 300 años del naufragio - 1708-2008 (Bogotá, Instituto de Estudios del Ministerio Público).

Oxman, Bernard H. (1982): The Third United Nations Conference on the Law of the Sea: The Tenth Session", American Journal of International Law, vol. 76(1): pp. 1-6.

Oxman, Bernard H. (1985): "From Cooperation to Conflict: The Soviet Union and the United States at the Third Conference on the Law of the Sea" en McKerman, Donald L. (ed.) Lectures in Marine Affairs (Seattle, Washington Grant Publications).

Ringbom, Henrik (2015): Jurisdiction over Ships: Post-UNCLOS Developments in the Law of the Sea (Leiden, Boston, Brill Nijhoff).

RoAch, J. Ashley (2012): "Warships, Sunken”, The Max Planck Encyclopedia of Public International (Oxford, Oxford University Press).

Rothwell, Donald D. et al. (2015): The Oxford Handbook of the Law of the Sea, (Oxford, Oxford University Press).

Sarmiento-Lamus, Andrés (2016): "Impacto e implementación en Colombia de la decisión de fondo de la Corte Internacional de Justicia en el Diferendo Territorial y Marítimo 
(Nicaragua c. Colombia)", Anuario Mexicano de Derecho Internacional, vol. XVI: pp. 401-423;

SMith Robert \& TAFT George (2000): "Legal Aspects of the Continental Shelf", en Cook, Peter J. y Carleton, Chris M. (Edit.), Continental Shelf Limits: The Scientific and Legal Interface (New York, Oxford University Press).

ScovazzI, Tullio (2000): “The Evolution of International Law of the Sea: New Issues, New Challenges" en Collected Courses of the Hague Academy of International Law.

Scovazzi, Tullio \& Garabello, Roberta (2003): The Protection of the Underwater Cultural Heritage Before and After the 2001 UNESCO Convention (Leiden, Boston, Martinus Nijhoff Publishers).

Talmon, Stefan (2015): “Determining Customary International Law: The ICJ’s Methodology between Induction, Deduction and Assertion", EJIL, vol. 26 (2): pp. 417-443.

Tladi, Dire (2016): First report on jus cogens, A/CN.4/693. Disponible en http://legal. un.org/docs/?symbol=A/CN.4/693. Fecha de consulta : 10 de diciembre de 2016.

SuÁrez, S.V. (2013): 'The Commission on the Limits of the Continental Shelf and its Function to Provide Scientific and Technical Advice', Chicago Journal of International Law, vol. 339 (12): pp. 345.

TanaKa, Y. (2012): The International Law of the Sea (Cambridge, New York, Melbourne, Madrid, Cape Town, Singapore, Sao Paulo, Mexico City, Cambridge University Press).

Uchkunova, Inna (2016), "The International Court of Justice: Delimitation of a Continental Shelf Extending Beyond 200 Nautical Miles", Kluwer Arbitration Blog. Disponible en http://kluwerarbitrationblog.com/2016/08/25/international-court-justice-delimitation-continental-shelf-extending-beyond-200-nautical-miles/. Fecha de consulta: 13 de enero de 2017.

United Nations Document (1982): A/CONF.62/RS.189, pp. 66, Párr. 251 “189th Plenary Meeting" (Statement of The Representative of Colombia).

United Nations Document (1982): A/CONF.62/L.78.

Uribe-Vargas, Diego (2001): Mares de Colombia: la acción diplomática que duplicó el territorio nacional (Bogotá, Universidad Jorge Tadeo Lozano).

Vega-Barbosa, Giovanny (2016): "The Admissibility of a Claim of Continental Shelf Rights Beyond 200nm Before an International Tribunal Absent a Recommendation by the CLCS: A Few Words About the ICJ's 2016 Judgment in Nicaragua v. Colombia", EJIL Talk. Disponible en https://www.ejiltalk.org/the-admissibility-of-a-claim-ofcontinental-shelf-rights-beyond-200-nm-before-an-international-tribunal-absent-arecommendation-by-the-clcs-a-few-words-about-the-icjs-2016-judgment-in-nicara/. Fecha de consulta: 22 enero de 2017.

Vega-Barbosa, Giovanny, et al (2014): "Traducción del Fallo de la Corte Internacional de Justicia en el caso del "Diferendo Marítimo" (Perú c. Chile). Decisión sobre el Fondo", Anuario Colombiano de Derecho Internacional, vol. 7: pp. 189-295.

Vega-Barbosa, Giovanny, et al (2015): "La delimitación marítima en el contexto de la desaparición del territorio estatal como consecuencia del cambio climático: análisis de los problemas jurídicos procedimentales y sustanciales de un escenario ya no tan hipotético", Revista Ius et Praxis, vol. 2: pp. 399 - 400. 
Vega-Barbosa, Giovanny (2017): "The Admissibility of Outer Continental Shelf Delimitation Claims Before the ICJ Absent a Recommendation by the CLCS”, Ocean Development \& International Law.

VIGNI, Patrizia (2013): “The enforcement of underwater cultural heritage by courts", Enforcing International Cultural Heritage Law (Oxford, Oxford University Press).

Whomersley, C. (2016): "The South China Sea: The Award of the Tribunal in the Case Brought by Philippines against China - A Critique", Chinese Journal of International Law, vol. 15 (2): pp. 239-264.

Wood, Michael (2016), Fourth report on identification of customary international law, A/ CN.4/695, pp. 10. Disponible en http://legal.un.org/docs/?symbol=A/CN.4/695. Fecha de consulta: 10 de febrero de 2017.

XueXiA, Liao (2017): "Evaluation of Scientific Evidence by International Court and Tribunals in the Continental Shelf Delimitation Cases", Ocean Development \& International Law, vol. 48 (1): pp. 4.

\section{JURISPRUDENCIA CITADA}

Corte Constitucional de Colombia. Sentencia C-915 de 2010. M.P. Humberto Sierra Porto.

Corte Constitucional de Colombia. Sentencia C-572 de 2014. M.P. Gabriel Eduardo Mendoza Martelo.

Estados Unidos: ODYSSEY MARINE V. UNIDENTIFIED SHIPWRECKED Vessel, 657F.3d (2011).

Estados Unidos: R.M.S. TITANIC, INC. V. HAVER, 171F3d (1999).

Estados Unidos: SEA HUNT V. UNIDENTIFIED SHIPWRECKED VESSEL, 221F.3d634 (2000).

Estados Unidos: TREASURE SALVORS, INC. V UNIDENTIFIED WRECKED AND ABANDONED SAILING VESSEL, 569F.2d (1978).

Estados Unidos: Guevara V. PERU, 468 F.3d (2006).

Corte Internacional de Justicia: Fisheries case, Judgment of December 18th, 1951: I.C.J. Reports 1951.

Corte Internacional de Justicia: Continental Shelf (Libyan Arab Jamahiriya/Malta), Judgment, I.C.J. Reports 1985, p. 13, p. 33, párr. 34.

Corte Internacional de Justicia: Maritime Delimitation and Territorial Questions between Qatar and Bahrain (Qatar v. Bahrain), Merits, Judgment, I.C.J. Reports 2001.

Corte Internacional de Justicia: Maritime Delimitation in the Indian Ocean (SOMALIA V. KENIA), Preliminary Objections, Judgment, 2 February 2017.

Corte Internacional de Justicia: Maritime Dispute (PERU v. CHILE), Judgment, I.C.J. Reports 2014.

Corte Internacional de Justicia: North Sea Continental Shelf, Judgment, I.C.J. Reports 1969.

Corte Internacional de Justicia: Territorial and Maritime Dispute (NICARAGUA V. COLOMBIA), Rejoinder of Colombia 2010.

Corte Internacional de Justicia: Territorial and Maritime Dispute (NICARAGUA v. CoLOMBIA), Judgment, I.C.J. Reports 2012. 
Corte Internacional de Justicia: Question of the Delimitation of the Continental Shelf between Nicaragua and Colombia beyond 200 nautical miles from the Nicaraguan Coast (NICARAGUa V. COlOMbIa), Judgment, Preliminary Objections, 17 March 2016.

Corte Permanente de Arbitraje: Island of Palmas Case (THE NETHERLANDS V. UNITED StATES OF AMERICA) Award of April 4, 1928, RIAA, Vol. II. (1949).

Corte Permanente de Arbitraje: The South China Sea Arbitration (PhILIPPINES V. CHINA) Award of July 12, 2016.

Colombia: Sala de Casación Civil de la Corte Suprema de Justicia. Sentencia de fecha 5 de julio de 2007. Radicado No. 08001-3103-010-1989-09134-01. M.P. Carlos Ignacio Jaramillo Jaramillo.

\section{DOCUMENTOS ELECTRÓNICOS}

Comunicado de prensa (16/11/2016): Participación de Colombia en el caso Supuestas Violaciones de Derechos Soberanos y Espacios Marítimos en el Mar Caribe (Nicaragua v. Colombia)" Disponible en http://www.cancilleria.gov.co/en/newsroom/publiques/ comunicado-prensa-participacion-colombia-caso-supuestas-violaciones-derechos. Fecha de consulta: 10 de diciembre de 2016.

El Espectador (4/12/2015): Santos anuncia hallazgo del Galeón San José”. Disponible en http://www.elespectador.com/noticias/nacional/santos-anuncia-hallazgo-del-galeon-sanjose-articulo-603615. Fecha de consulta: 1 de abril de 2017.

Miles. Edward (1998): "Global Ocean Politics - The Decision Process at the Third United Nations Conference on the Law of the Sea 1973-1982. p. 36 y ss. Disponible en http:// www.un.org/Depts/los/reference_files/status2010.pdf. Fecha de consulta: 1 de abril de 2107.

Ocean Law News (30/3/2017): “Changing Strategies for the LOS Convention”. Disponible en http://us6.campaign-archive2.com/?u=f8f44615fa\&id=2ef999032d. Fecha de consulta: 3 de marzo de 2017.

Senate Treaty Document 103-39 (2012). Written Testimony of Hillary Rodham Clinton Secretary of State on Accession to the 1982 Law of the Sea Convention and Ratification of the 1994 Agreement amending Part XI of the Law of the Sea Convention”, pp. 9-10 Disponible en www.foreign.senate.gov/imo/media/doc/REVISED_Secretary_Clinton_ Testimony.pdf. Fecha de consulta: 16 de marzo de 2017.

Revista Semana (5/12/2015): "El presidente Santos celebró el hallazgo del galeón San José", Disponible en http://www.semana.com/nacion/articulo/presidente-juan-manuelsantos-celebra-hallazgo-del-galeon-san-jose/452344-3. Fecha de consulta: 10 de enero de 2017.

US Department of State. "Law of the Sea Convention". Disponible en: https://www.state. gov/e/oes/lawofthesea/. Fecha de consulta: 10 de enero de 2017.

US Department of State. "Protection of Sunken Warships, Military Aircraft and Other Sunken Government Property. Disponible en: http://www.gpo.gov/fdsys/pkg/FR-200402-05/html/04-2488.htm (February 2004). Fecha de consulta: 1 de abril de 2017. 


\section{NORMAS CITADAS}

Acuerdo relativo a la aplicación de la Parte XI de la Convención de las Naciones Unidas sobre el Derecho del Mar [1836 UNTS 41].

Aст No. 411 (22/5/1996) on Exclusive Economic Zones.

Convención de Naciones Unidas sobre el Derecho del Mar [1833 UnTS 3].

Convención de Viena sobre el Derecho de los Tratados [1155 UNTS 331].

Convención Unesco sobre Patrimonio Cultural Subacuático de 2001.

Convenio Internacional sobre Salvamento Marítimo de 1989.

Decreto No. 29 (10/01/1984), "Por el cual se crea la Comisión de Antigüedades Náufragas".

DeCreto No. 12 (10/1/1984).

Decreto Ley 2349 de 1971.

Decreto No. 1946 (9/9/2013) "Por medio del cual se reglamenta la Ley 47 de 1993 en lo concerniente al mar territorial, la zona contigua, algunos aspectos de la plataforma continental de los territorios insulares colombianos en el mar Caribe occidental y a la integridad del departamento archipiélago de San Andrés, Providencia y Santa Catalina”.

Decreto No. 1119 (17/6/2014) "Por el cual se modifica y adiciona el Decreto Número 1946 de 9 de septiembre de 2013".

Ley Federal del Mar (8/1/1986) de los Estados Unidos Mexicanos.

Ley No. 1675 (30/7/2013) "Por medio de la cual se reglamentan los artículos 63, 70 y 72 de la Constitución Política de Colombia en lo relativo al Patrimonio Cultural Sumergido".

LeY No. 10 (04/08/1978) "Por medio de la cual dictan normas sobre mar territorial, zona económica exclusiva, plataforma continental, y se dictan otras disposiciones".

ResoluCión No. 0066 (4/2/1981).

REsoluCión No. 0048 (29/1/1980).

Oceans Act (S.C. 1996, c. 31): Oceans Act of Canada. 